\title{
Nanosecond laser ablation of a metallic glass in water: a high time-resolved imaging study
}

\author{
X. Song , X. Q. Wu , K. L. Xiao , C. Li , H. Y. Wang \& M. Q. Jiang
}

To cite this article: X. Song , X. Q. Wu , K. L. Xiao , C. Li , H. Y. Wang \& M. Q. Jiang (2020): Nanosecond laser ablation of a metallic glass in water: a high time-resolved imaging study, Philosophical Magazine, DOI: 10.1080/14786435.2020.1791369

To link to this article: https://doi.org/10.1080/14786435.2020.1791369

\section{+ View supplementary material $₫$}

曲 Published online: 20 Jul 2020.

Submit your article to this journal

山 Article views: 60

Q View related articles ¿

View Crossmark data $\nearrow$ 


\title{
Nanosecond laser ablation of a metallic glass in water: a high time-resolved imaging study
}

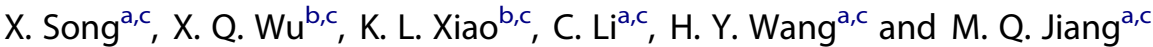

aState Key Laboratory of Nonlinear Mechanics, Institute of Mechanics, Chinese Academy of Sciences,

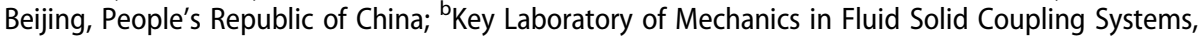
Institute of Mechanics, Chinese Academy of Sciences, Beijing, People's Republic of China; 'School of Engineering Science, University of Chinese Academy of Sciences, Beijing, People's Republic of China

\begin{abstract}
We use a state-of-the-art, ultrafast camera to probe the nanosecond pulse laser ablation of a metallic glass target in water. The laser-induced plasma of metallic glass is captured for the first time. We further observe a series of shock waves in water and the cavitation bubble on the target surface. The shock waves inherit the rapid expansion of the plasma, satisfying the momentum conservation. But the cavitation bubble stems from the nucleation of the surrounding water due to the heating of the plasma. We find that the bubble growth deviates from the RayleighPlesset theory. The primary reason for this deviation is the effect of ablation products due to the temporal overlap between the explosive boiling and the bubble dynamics. Our findings provide a fundamental understanding of underwater laser ablation of metallic glasses, which is an important step to forward such ablation-related applications.
\end{abstract}

\section{ARTICLE HISTORY}

Received 27 May 2020

Accepted 30 June 2020

\section{KEYWORDS}

Metallic glasses; nanosecond laser ablation; plasma; shock wave; cavitation bubble

\section{Introduction}

Interaction between high-energy laser and metallic glasses continues to fascinate researchers because of the engineering and scientific significance [1-10]. During such interactions, laser-induced plasma plays an important role. For example, the strength and plasticity of metallic glasses can be effectively tuned by plasma-generated shock wave (SW), which is known as laser shock peening [11-13]. Irradiated by nanosecond pulse lasers, the surfaces of metallic glasses usually exhibit the Kelvin-Helmholtz instability in air and the Saffman-Taylor fingering in water [14-16]. It has been accepted that these unique hydrodynamic instabilities are ascribed to the strong interaction between the expanding plasma plume and the molten layer. During underwater laser ablations, the cavitation bubble is frequently observed to take place on the target surface, which provides 
an ideal place to produce nanoparticles with desirable structures and properties [17-21]. By considering the plasma-induced nucleation of the surrounding water, the cavitation bubble dynamics has been quantitatively described for a metallic glass target [22]. More importantly, the existence of plasma will change the transfer of laser energy into the target, and thus affects the thermal ablation processes (e.g. explosive boiling) [23-25]. Despite its paramount importance, the laser-induced plasma has not yet been captured experimentally for the targets of metallic glasses. It is a fortiori unknown when and how the plasma excites SWs and cavitation bubbles.

In this work, we conduct in situ, high time-resolved observations of nanosecond pulse laser ablation on a typical Zr-based bulk metallic glass in water. The laser-induced plasma and the plasma-excited SWs and cavitation bubble are successfully observed, and the underlying mechanisms for these physical phenomena are revealed as well.

\section{Experiment and method}

We adopted a typical $\mathrm{Zr}_{41.2} \mathrm{Ti}_{13.8} \mathrm{Cu}_{12.5} \mathrm{Ni}_{10.0} \mathrm{Be}_{22.5}$ (Vitreloy 1) metallic glass as the target material. The targets were cut into rectangular specimens $(10 \times 10 \times 3.6$ $\mathrm{mm}^{3}$ ), and their glassy nature was confirmed by X-ray diffraction and differential scanning calorimetry. Single-pulse ablation experiments were conducted by using a Nd:YAG laser (wavelength $1064 \mathrm{~nm}$ and pulse energy $1.9 \mathrm{~J}$ ), and the schematic illustration of the experimental setup is shown in Figure 1. The

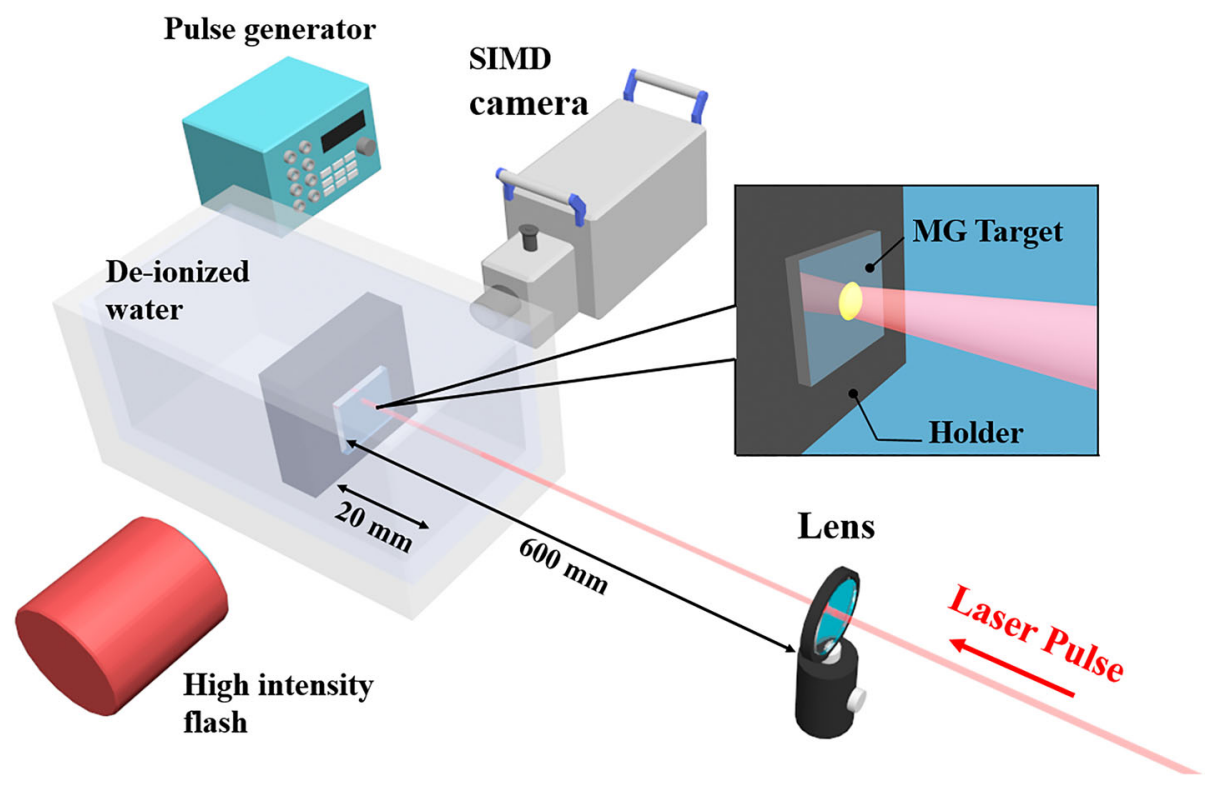

Figure 1. Schematic illustration of the experimental setup for the nanosecond laser ablation of the Vitreloy 1 target in water. 
temporal profile of the laser pulse approximates to a Gaussian distribution and its full width at half maxima (FWHM), i.e. the pulse width, is about $10 \mathrm{~ns}$. The target was submerged in filtered and de-ionized water. The incident laser beam was normal to the target surface. The laser energy intensity $I_{\text {Inc }}$ was fixed to be about $3.7 \mathrm{GW} / \mathrm{cm}^{2}$. An ultrafast camera (Specialised Imaging SIMD) was used to in situ record the physical processes associated with the laser ablation. This camera can consecutively record 16 images with a size of $1280 \times 960$ pixels at all frame rates up to 1,000,000,000 fps. The trigger of the camera and the laser pulse was controlled by a pulse generator (BNC 575) with the time accuracy of $10 \mathrm{fs}$. The time window for each record equals 16 times the inverse of selected frame rate. The delay technique of trigger time was used to overlap the time windows, thus permitting longer time records. All images were illuminated by multiple head high-intensity flash system (Specialised Imaging AD500).

\section{Results and discussion}

\subsection{Laser-induced plasma}

Figure 2 shows the recorded images of the initial plasma from the Vitreloy 1 target irradiated by the nanosecond pulse laser in water. The first image ( $0 \mathrm{~ns})$ corresponds to the start point of the FWHM of laser pulse. During the first $10 \mathrm{~ns}$, i.e. the pulse width, the bright plasma expands dramatically with a velocity of about $44-50 \mathrm{~km} / \mathrm{s}$ along the target surface. In contrast, the expansion velocity along the laser path direction is only about $1.5 \mathrm{~km} / \mathrm{s}$, which is far below than that in the air [26]. The constraint of water greatly limits the outward expansion of the plasma. The brightness enhancement of the plasma implies the dramatic increase in its temperature and pressure within the $10 \mathrm{~ns}$ pulse width. The peak temperature and pressure can be as high as $10^{4} \mathrm{~K}$ and $10^{9} \mathrm{~Pa}$, respectively [27]. In this stage, we also observe the laser-induced plasmatization of

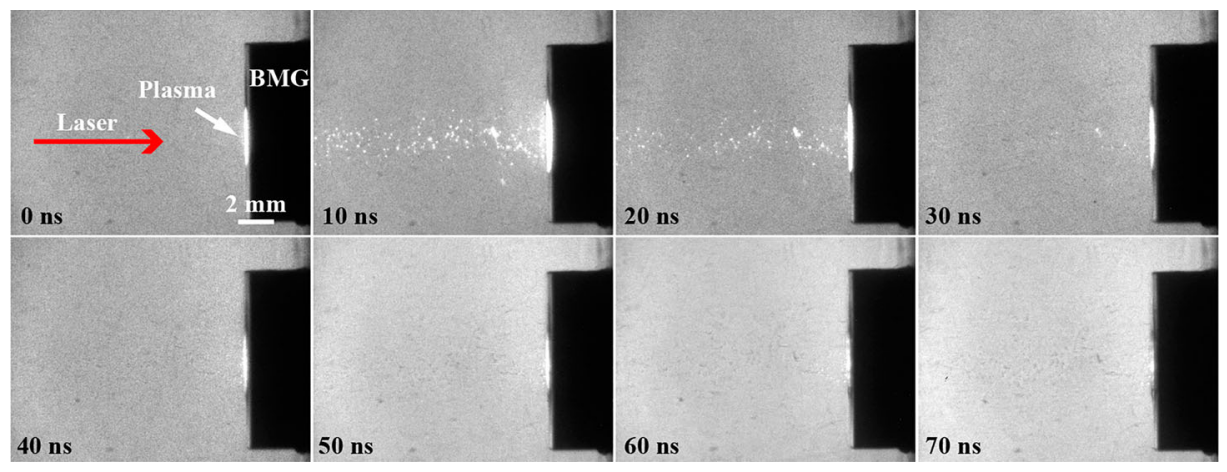

Figure 2. Experimental capture of the plasma induced by the laser irradiation on the Vitreloy 1 target in water. Bright spots are observed on the laser path, indicating the laser-induced plasmatization of water. 
surrounding water, shown as many bright spots on the incident laser path. After about 10 ns, the plasma begins to cool accompanied by the decrease in its brightness. Eventually, the temperature, pressure and size of the plasma will reach a steady state at tens of nanoseconds after the laser pulse termination [22, 27]. This means that even without the further input of laser pulse energy, the high temperature and pressure plasma will still 'pressurize' and 'warm' both target and surrounding water, giving rise to other physical processes [28, 29]. To the best of our knowledge, this is the first time one can experimentally capture the laser-induced plasma of a metallic glass.

\subsection{Shock wave}

The rapidly expanding plasma usually imposes a pulse-like impact on the bothside constraints. Following the impact, an outward compressive SW and an inward one are generated simultaneously, and then propagate into the water and the target, respectively. Figure 3 shows the propagation of the SWs in water due to the present laser ablation. The corresponding full-process images are presented in the supplementary material, Figure S1. The first image (at $\sim 100$ ns after the laser irradiation) captures the critical process when the outward primary SW is just separated from the plasma edge. Before this separation time (100 ns), the SW is overlapped with the plasma, and not visible until it travels beyond the plasma. This primary SW initially looks like a shadow around the illuminant plasma, and then propagates into the water with a planar front

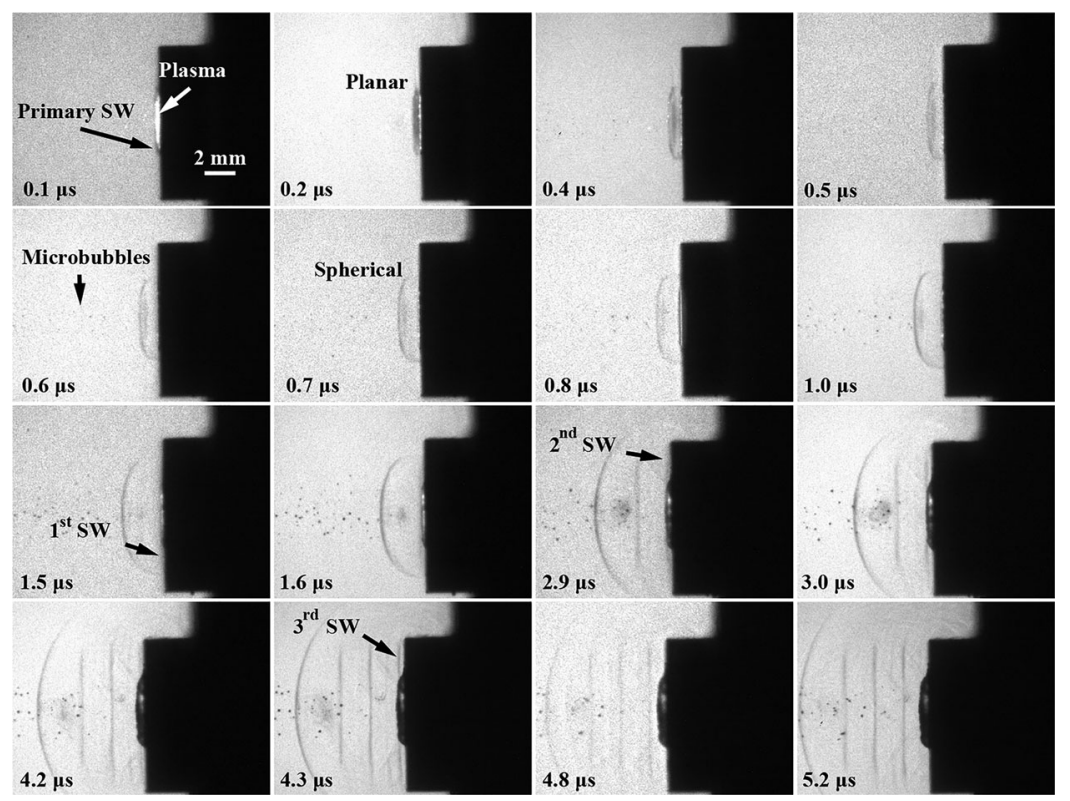

Figure 3. Spatiotemporal evolution of the plasma-excited shock waves in water. The time records take the first image of Figure 2 as the reference point. 
before $\sim 600$ ns. After that, the plane wave gradually changes into an ideal spherical wave. This is because, with the increase of propagation distance, the plasma can be approximately regarded as a point source of blast. In addition to the primary SW, it is interesting to observe that a new SW with the planar front will appear every about $1.4 \mu \mathrm{s}$. During the current observation window of $5.2 \mu \mathrm{s}$, there appear three plane waves propagated from the target to the water. According to their appearance orders, these plane waves are numbered as $1^{\text {st }}, 2^{\text {nd }}$ and $3^{\text {rd }}$ SW, respectively.

We measure the propagation distances $D$ of the observed four SWs with time $t$, and further calculate their propagation velocities $V_{w}$ in water. The results are presented in Figure 4. For the primary SW, its initial $V_{w}$ is about $2.2 \mathrm{~km} / \mathrm{s}$ at $100 \mathrm{~ns}$ and then decays gradually toward the sound speed in water $(\sim 1.5 \mathrm{~km} /$ s) at the duration of $\sim 1 \mu \mathrm{s}$. This velocity decay process corresponds to the observed transition from the planar to the spherical front; see Figure 3. Obviously, the primary SW is initially supersonic, and its travel dynamics before $600 \mathrm{~ns}$ obeys the Taylor-Sedov theory [30, 31]: $D \sim t^{0.72}$, where the decay rate of 0.72 is close to that $(0.67)$ of an ideal plane wave. Based on the work of Pezeril et al. [32], the pressure of the outward primary SW is estimated to be $\sim 0.74 \mathrm{GPa}$ at the separation time $(100 \mathrm{~ns})$, and before that its average pressure is about $3.57 \mathrm{GPa}$. The detailed calculation is provided in the supplementary material. These pressure values of the primary SW agree well with the plasma pressure calculated previously [22]. Such an agreement implies that the plasma-induced SW is nearly an adiabatic process dominated by the momentum conversion.

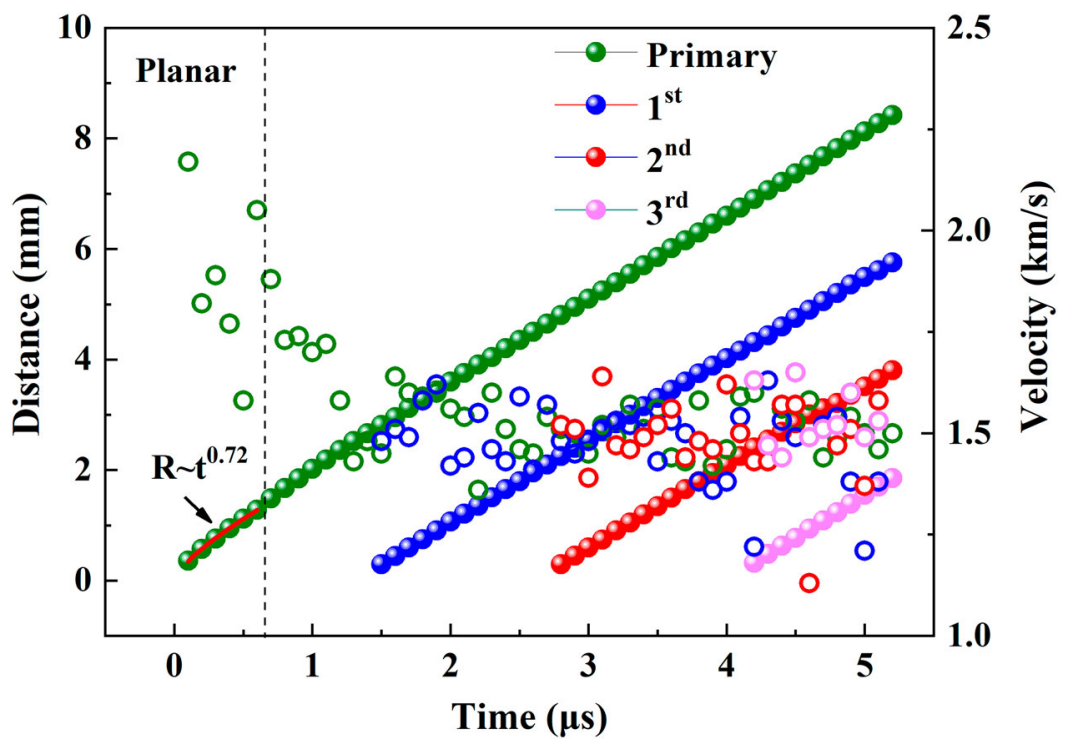

Figure 4. Measured propagation distances (filled balls) of the shock waves from the target surface and the calculated propagation velocities (open circles) of these shock waves in water. 
For the subsequent three plane waves in water, their propagation velocities keep a constant of about $1.5 \mathrm{~km} / \mathrm{s}$, indicating the nature of the sound wave. As mentioned above, the interval time of the four SWs is almost the same as $\sim 1.4 \mu \mathrm{s}$, from which we can infer two important facts. First, the plasmainduced compressive wave propagated into the target is an elastic (or sound) wave. It means that the initial stress amplitude of the compressive wave is less than the Hugoniot elastic limit (HEL) of the target. The peak pressure of the inward SW is calculated to be $\sim 3.51 \mathrm{GPa}$ in the supplementary material. This pressure is indeed lower than the reported HEL (6-7 GPa) of Vitreloy 1 [33]. It must be pointed out that the pressure between the plasma, the outward and inward SWs resides in a momentum balance state during the initial $100 \mathrm{~ns}$. Second, the $1^{\text {st }}, 2^{\text {nd }}$ and $3^{\text {rd }}$ SWs are a result of the reflection and transmission of the inward SW at the interfaces of target-holder and target-water, respectively. Considering the longitudinal sound velocity $(\sim 5174 \mathrm{~m} / \mathrm{s})$ of the Vitreloy 1 , the interval time $(\sim 1.4 \mu \mathrm{s})$ matches well with that an elastic wave takes one round trip within the target thickness $(3.6 \mathrm{~mm})$.

Figure 5 presents the $t$ - $X$ (time versus distance) diagram that details how the inward SW propagates in the target and two-side media. When an elastic wave propagates to a non-free interface, it converts into a reflected wave and a transmitted wave. The transmitted wave enters the adjacent medium, but the reflected

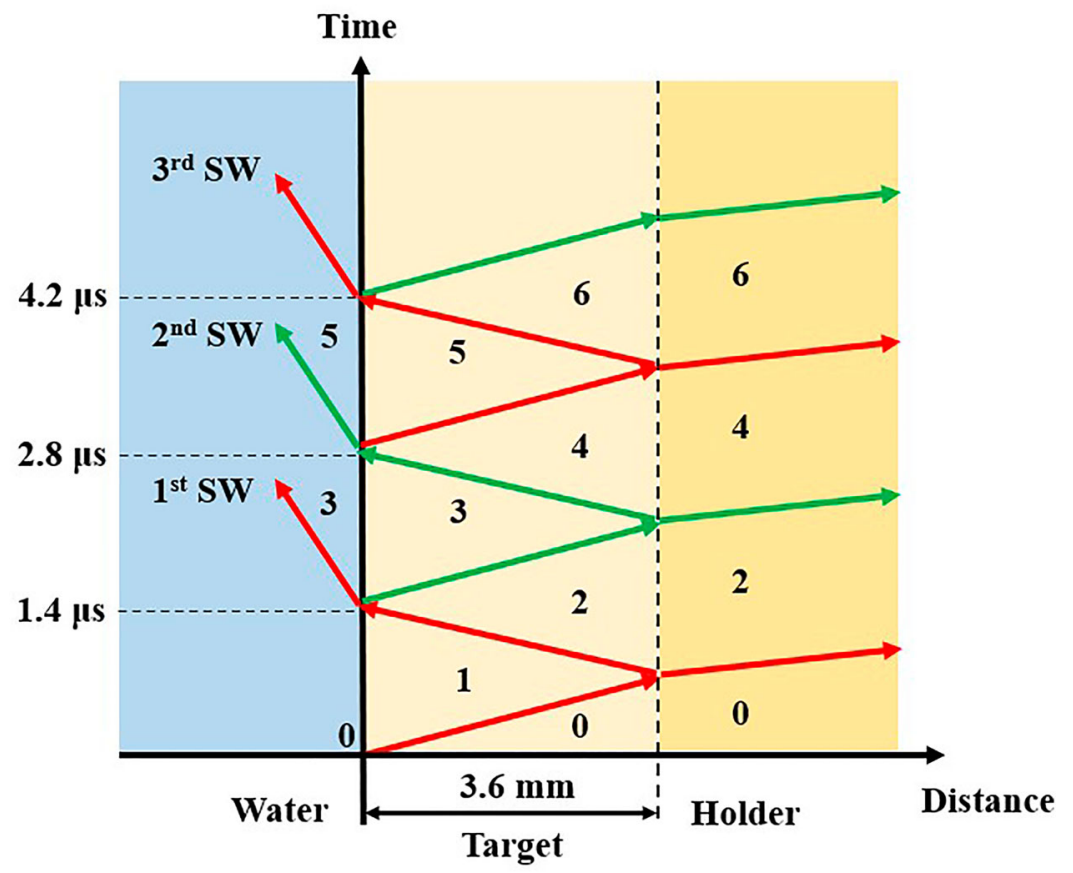

Figure 5. Time versus distance $(t-X)$ diagram that illustrates how the inward shock wave propagates in the metallic glass target and induces the periodic plane waves in water. The red arrows indicate the compression waves and the green arrows indicate the tensile waves. The ordered numbers $(0-6)$ indicate the different stress states of the three media. 
wave travels backward. In fact, the observed three plane waves (Figure 3) are the transmitted waves at the target-water interface when the inward SW has completed a reciprocating propagation inside the target. For the k-th plane wave, it has experienced $\mathrm{k}$ and $\mathrm{k}-1$ reflections at the target-holder and target-water interfaces, respectively, and 1 transmission at the target-water interface. The detailed calculation and description of the stress state of these plane waves are provided in the supplementary material. We find that these plane waves appear alternately as a compression wave and a tensile one and their stress amplitudes decrease rapidly with increasing reflection.

\subsection{Cavitation bubble}

At about 560 ns after the laser irradiation, we identify the nucleation of a primary cavitation bubble on the target surface, which is shown in Figure 6. The bubble nucleation results from the close contact between the hot plasma and the surrounding water. The latter will reach a supercritical state and then adiabatically expands to an inflating bubble [25]. It is worth noting that some microbubbles form on the laser path almost at the same time (Figure 3), although their sizes are much smaller than the primary bubble. Obviously, these microbubbles are nucleated by the plasmas of water. After nucleation, the primary bubble begins to grow. The growth dynamics in terms of the bubble radius $R(t)$ is usually described by the Rayleigh-Plesset (RP) equation $[22,34,35]$ :

$$
\rho_{W}\left(R \ddot{R}+\frac{3}{2} \dot{R}^{2}\right)=P_{B}(t)-P_{\infty}-2 \frac{\sigma}{R}-4 \frac{\eta \dot{R}}{R},
$$

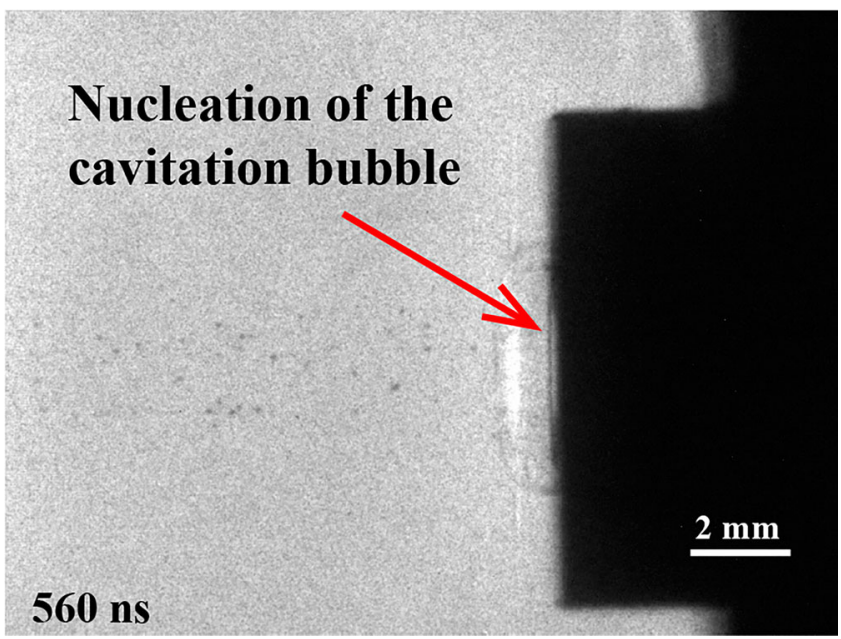

Figure 6. The plasma-induced nucleation of the cavitation bubble on the target surface. The time (560 ns) takes the first image of Figure 2 as the reference point. 
where $P_{B}(t), \rho_{W}, P_{\infty}, \sigma$ and $\eta$, respectively, represent the internal pressure of the bubble, the density, the far-field pressure, the surface tension and the dynamic viscosity of the surrounding water. We, as usual, measure the bubble radius $R(t)$ as the maximum perpendicular distance from the front of the bubble to the target surface. The internal pressure of bubble $P_{B}(t)$ is determined by its radius:

$$
P_{B}(t)=P_{B}(0)\left(R_{0} / R(t)\right)^{3 \varphi},
$$

where $P_{B}(0), R_{0}$ and $\varphi$ are the initial internal pressure and radius of the bubble, and the adiabatic exponent of the vapour, respectively. Here, $P_{B}(0)$ is equal to the plasma pressure (about $100 \mathrm{kPa}$ ) at the nucleation time (560 ns) [22]. We obtain $R_{0}$ based on the volume conservation:

$$
4 \pi R_{0}^{3} / 3=H_{0} \pi\left(\frac{D_{0}}{2}\right)^{2} .
$$

We regard the initial bubble in Figure 6 as a flat cylinder, where $H_{0}$ and $D_{0}$ are the thickness and diameter of the cylinder, respectively. The parameter $\dot{R}_{0}$ is determined by fitting the slope of the initial bubble radius with the time. Relevant parameters for the calculation of the bubble dynamics are summarised in the supplementary materials, Table S2. Figure 7 shows the comparison between the theoretical prediction and the experimental measurements (see the supplementary materials, Figure S2).

From Figure 7, we see that the early stage (before $300 \mu$ s) of the bubble growth is roughly consistent with the theoretical prediction, but during the first tens of microseconds the experimental data are overestimated by the theory. Particularly after $300 \mu \mathrm{s}$, the data gradually deviate downward from the prediction. We think that this deviation is ascribed to the influence of the ablation products and the limited size of the target surface. Figure 8 presents the results about the initial expansion of the cavitation bubble at $2 \mu$ s frame interval. We find that the microbubbles nucleated by the plasmas of water completely disappear from the laser path after $18 \mu \mathrm{s}$. However, a cloud of shadow appears again outside the bubble at $24 \mu \mathrm{s}$, as indicated by the black arrow in Figure 8 . The shadow exhibits a dispersed distribution without significant movement. We believe that the shadow consists of microbubbles, but they are induced by the high-temperature ablation products suspended in water. These ablation products should result from the superheating of the Vitreloy 1 target and resultant explosive boiling. According to our previous study $[20,24]$, the explosive boiling takes place at $\sim 20$ ns after the end of the laser pulse and this process can last for tens of microseconds. Obviously, the observed microbubbles just appear within this temporal range, which provides evidence for the occurrence of the explosive boiling. But at the current time-resolution of $2 \mu \mathrm{s}$, the explosive boiling process is not captured. A possible reason is that the high photosensitivity threshold of used imaging 


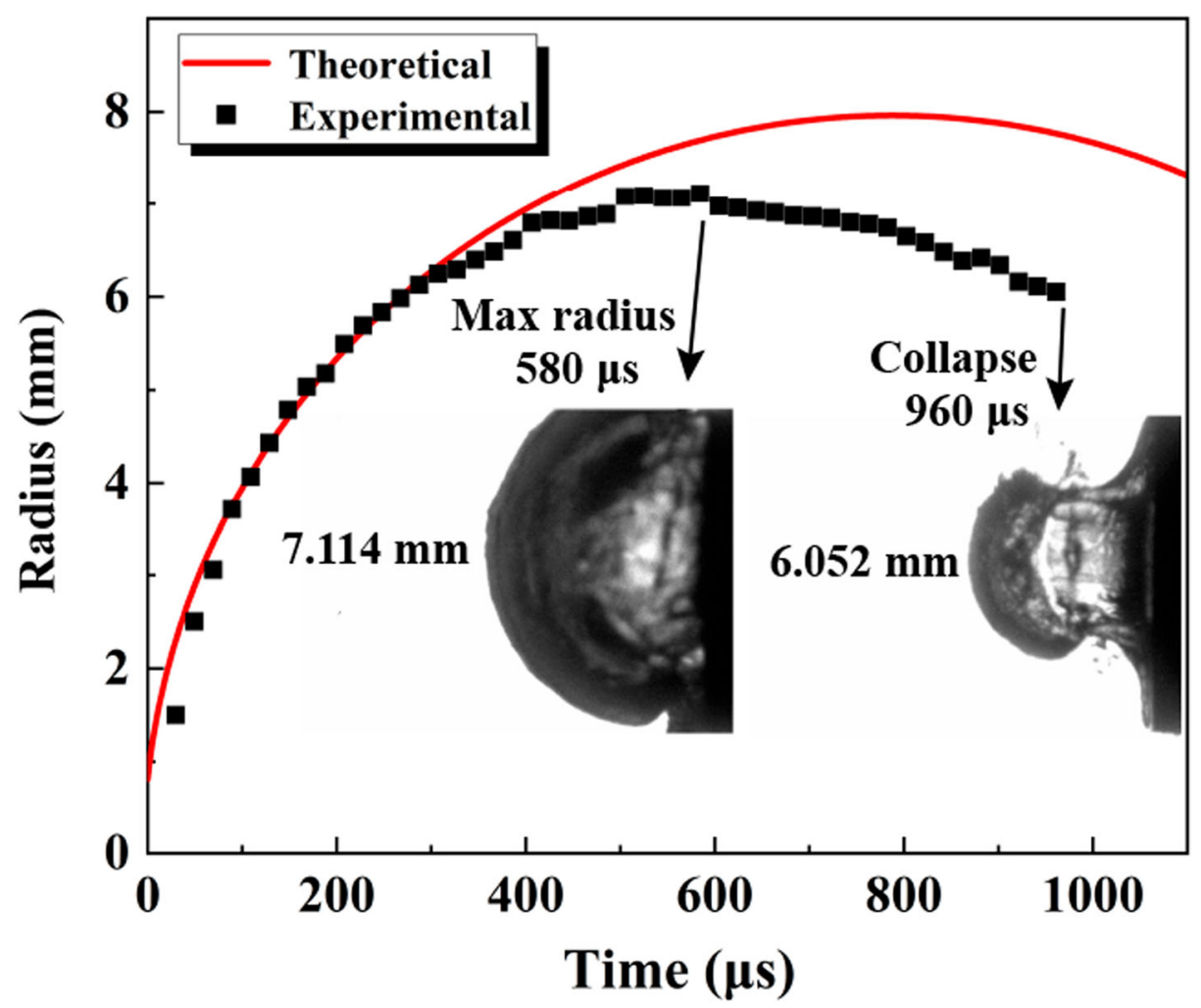

Figure 7. Comparison between the theoretical prediction (solid line) and the experimental measurement (filled squares) of the bubble dynamics. The insets show the bubble with the maximum radius and its collapse.

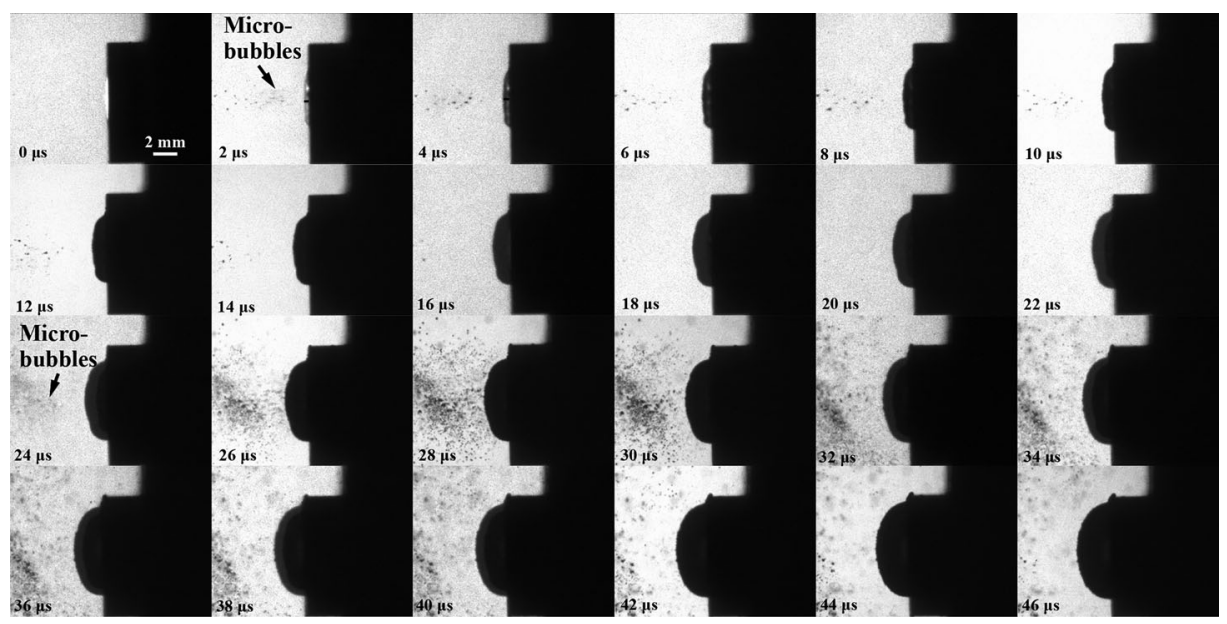

Figure 8. Initial expansion stage of the cavitation bubble on the target surface at $2 \mu$ interval. The microbubbles are observed outside the bubble when it grows. 
setup exactly ignores the luminance of these high-temperature matters. However, after the ablation products enter the water, they will excite a shadelike microbubble nucleation of the surrounding water.

The temporal overlapping between the explosive boiling and the bubble dynamics incurs the prediction deviation because the traditional RP theory does not take into account the effect of mass exchange for bubble [22, 35]. From the observation of the spatial location of the microbubbles in Figure 8 , some of ablation products directly enter the water before the bubble nucleation. These suspended products increase the density and viscosity of the surrounding water, thus suppressing the bubble growth. This suppression effect is particularly obvious under the multi-pulse laser ablation [22, 36]. The rest of the ablation products are trapped inside the primary bubble, which slows down the bubble growth and meanwhile speeds up its collapse. Actually, we indeed did not observe the rebound of the bubble; see the supplementary materials, Figure S2. The different ways via which ablation products enter the water have been evidenced by the final nanoparticles with different structures and compositions [20].

The secondary reason for the deviation comes from the limited size of the target surface. It is well accepted that the large-size target surface can provide the stable support for the bubble growth, which is an important precondition for using the Rayleigh-Plesset theory. In the present work, the size of the target surface is $10 \times 10 \mathrm{~mm}^{2}$. From the observations of the bubble growth in the supplementary materials, Figure S2, we find that the bottom size of the bubble reaches the maximum $(10 \mathrm{~mm})$ at about $200 \mu \mathrm{s}$, and then does not increase anymore without the further support from the target surface. After about $300 \mu \mathrm{s}$, the bubble bottom starts to shrink, which exactly corresponds to the deviation of the perpendicular radius downward from the theoretical prediction; see Figure 7. Therefore, the unstable bottom of the bubble also contributes to its earlier collapse.

\section{Conclusions}

In summary, the physical processes associated with the underwater laser ablation of a Vitreloy 1 metallic glass were directly observed by a very high timeresolved camera. The laser-induced plasma was captured to initiate within the pulse-width time. The rapidly expanding plasma excites the outward and inward SWs that propagate into the water and the target, respectively. The outward SW will separate from the plasma and evolve from a planar supersonic to spherical sound wave. The inward SW is responsible for the repeated appearance of a series of planar sound waves in water. The nucleation of the cavitation bubble on the target surface was observed at $\sim 560$ ns after the pulse end, which is due to the thermal effect of the plasma. We reveal that there is a temporal overlapping between the explosive boiling and the growth dynamics of the bubble, 
and therefore the latter cannot be well described by the traditional RP theory. In addition, the limited size of target surface incurs the unstable bottom of the bubble, which also leads to its early collapse.

\section{Disclosure statement}

No potential conflict of interest was reported by the author(s).

\section{Funding}

This work was supported by the National Natural Science Foundation of China (NSFC) Basic Science Center for 'Multiscale Problems in Nonlinear Mechanics' (grant number 11988102), the NSFC (grant numbers 11972345, and 11790292), and the Youth Innovation Promotion Association of Chinese Academy of Sciences (grant number Y201704).

\section{References}

[1] T.J. Zhu, L. Lu, and L.Q. Yao, Excimer laser-induced transformation in laser ablated Pb (Zr0.52Ti0.48)O-3 amorphous thin films. Philos. Mag. 84 (2004), pp. 3729-3739.

[2] I. Quintana, T. Dobrev, A. Aranzabe, G. Lalev, and S. Dimov, Investigation of amorphous and crystalline Ni alloys response to machining with micro-second and picosecond lasers. Appl. Surf. Sci. 255 (2009), pp. 6641-6646.

[3] F. Ma, J. Yang, X. Zhu, C. Liang, and H. Wang, Femtosecond laser-induced concentric ring microstructures on Zr-based metallic glass. Appl. Surf. Sci. 256 (2010), pp. 3653-3660.

[4] S. Pauly, L. Loeber, R. Petters, M. Stoica, S. Scudino, U. Kuehn and J. Eckert, Processing metallic glasses by selective laser melting. Mater. Today 16 (2013), pp. 37-41.

[5] E. Williams and E.B. Brousseau, Nanosecond laser processing of Zr 41.2 Ti $13.8 \mathrm{Cu} 12.5$ Ni 10 Be 22.5 with single pulses. J. Mater. Process. Technol. 232 (2016), pp. 34-42.

[6] H. Huang, N. Jun, M.Q. Jiang, M. Ryoko, and J.W. Yan, Nanosecond pulsed laser irradiation induced hierarchical micro/nanostructures on Zr-based metallic glass substrate. Mater. Des. 109 (2016), pp. 153-161.

[7] E. Williams and N. Lavery, Laser processing of bulk metallic glass: A review. J. Mater. Process. Technol. 247 (2017), pp. 73-91.

[8] H. Huang, M.Q. Jiang, and J.W. Yan, Softening of Zr-based metallic glass induced by nanosecond pulsed laser irradiation. J. Alloys Compd. 754 (2018), pp. 215-221.

[9] L. Zhang and H. Huang, Micro machining of bulk metallic glasses: A review. Int. J. Adv. Manuf. Technol. 100 (2019), pp. 637-661.

[10] Y. Jiao, E. Brousseau, Q.Q. Han, H.X. Zhu, and S. Bigot, Investigations in nanosecond laser micromachining on the Zr52.8Cu17.6Ni14.8Al9.9Ti4.9 bulk metallic glass: Experimental and theoretical study. J. Mater. Process. Technol. 273 (2019), pp. 6-13.

[11] G.J. Wu, R. Li, Z.Q. Liu, B.Q. Chen, Y. Li, Y. Cai and T. Zhang, Induced multiple heterogeneities and related plastic improvement by laser surface treatment in $\mathrm{CuZr}$-based bulk metallic glass. Intermetallics 24 (2012), pp. 50-55.

[12] J. Fu, Y.H. Zhu, C. Zheng, R. Liu, and Z. Ji, Effect of laser shock peening on mechanical properties of Zr-based bulk metallic glass. Appl. Surf. Sci. 313 (2014), pp. 692-697.

[13] Y.F. Cao, X. Xie, J. Antonaglia, B. Winiarski, G.Y. Wang, Y.C. Shin, P.J. Withers, K.A. Dahmen, and P.K. Liaw, Laser shock peening on Zr-based bulk metallic glass and its effect on plasticity: Experiment and modeling. Sci. Rep. 5 (2015), pp. 10789.1-10789.8. 
[14] Y. Liu, M.Q. Jiang, G.W. Yang, Y.J. Guan, and L.H. Dai, Surface rippling on bulk metallic glass under nanosecond pulse laser ablation. Appl. Phys. Lett. 99 (2011), pp. 191902.1-191902.3.

[15] Y. Liu, M.Q. Jiang, G.W. Yang, J.H. Chen, Y.J. Guan and L.H. Dai, Saffman-Taylor fingering in nanosecond pulse laser ablating bulk metallic glass in water. Intermetallics 31 (2012), pp. 325-329.

[16] Y.H. Zhu, J. Fu, C. Zheng, and Z. Ji, Effect of nanosecond pulse laser ablation on the surface morphology of Zr-based metallic glass. Opt. Laser Technol. 83 (2016), pp. 21-27.

[17] A. De Giacomo, M. Dell'Aglio, A. Santagata, R. Gaudiuso, O. De Pascale, P. Wagener, G.C. Messina, G. Compagnini, and S. Barcikowski, Cavitation dynamics of laser ablation of bulk and wire-shaped metals in water during nanoparticles production. Phys. Chem. Chem. Phys. 15 (2013), pp. 3083-3092.

[18] J. Xiao, P. Liu, C.X. Wang, and G.W. Yang, External field-assisted laser ablation in liquid: An efficient strategy for nanocrystal synthesis and nanostructure assembly. Prog. Mater Sci. 87 (2017), pp. 140-220.

[19] L. Torrisi, M. Cutroneo, L. Silipigni, F. Barreca, B. Fazio, N. Restuccia, and L. Kovacik, Gold nanoparticles produced by laser ablation in water and in graphene oxide suspension. Philos. Mag. 98 (2018), pp. 2205-2220.

[20] X. Song, K.L. Xiao, X.Q. Wu, G. Wilde, and M.Q. Jiang, Nanoparticles produced by nanosecond pulse laser ablation of a metallic glass in water. J. Non-Cryst. Solids 517 (2019), pp. 119-126.

[21] N.A. Inogamov, V.A. Khokhlov, Y.V. Petrov, and V.V. Zhakhovsky, Hydrodynamic and molecular-dynamics modeling of laser ablation in liquid: from surface melting till bubble formation. Opt. Quantum Electron. 52 (2020), pp. 1-24.

[22] M.Q. Jiang, X.Q. Wu, Y.P. Wei, G. Wilde, and L.H. Dai, Cavitation bubble dynamics during pulsed laser ablation of a metallic glass in water. Extreme Mech. Lett. 11 (2017), pp. 24-29.

[23] B.J. Demaske, V.V. Zhakhovsky, N.A. Inogamov and I.I. Oleynik, Ablation and spallation of gold films irradiated by ultrashort laser pulses. Phys. Rev. B 82 (2010), pp. 064113.1-064113.5.

[24] M.Q. Jiang, Y.P. Wei, G. Wilde, and L.H. Dai, Explosive boiling of a metallic glass superheated by nanosecond pulse laser ablation. Appl. Phys. Lett. 106 (2015), pp. 021904.1021904.5.

[25] Y.V. Petrov, V.A. Khokhlov, V.V. Zhakhovsky, and N.A. Inogamov, Hydrodynamic phenomena induced by laser ablation of metal into liquid. Appl. Surf. Sci. 492 (2019), pp. 285-297.

[26] J. Wu, W.F. Wei, X.W. Li, S.L. Jia, and A.C. Qiu, Infrared nanosecond laser-metal ablation in atmosphere: Initial plasma during laser pulse and further expansion. Appl. Phys. Lett. 102 (2013), pp. 164104.1-164104.4.

[27] D. Marla, Y. Zhang, J.H. Hattel, and J. Spangenberg, Modeling of nanosecond pulsed laser processing of polymers in air and water. Modell. Simul. Mater. Sci. Eng. 26 (2018), pp. 1-21.

[28] J.H. Yoo, S.H. Jeong, X.L. Mao, R. Greif, and R.E. Russo, Evidence for phase-explosion and generation of large particles during high power nanosecond laser ablation of silicon. Appl. Phys. Lett. 76 (2000), pp. 783-785.

[29] J.J. Yoh, H. Lee, J. Choi, K.-C. Lee, and K.-H. Kim, Ablation-induced explosion of metal using a high-power Nd:YAG laser. J. Appl. Phys. 103 (2008), pp. 043511.1-043511.6.

[30] S.H. Jeong, R. Greif, and R.E. Russo, Propagation of the shock wave generated from excimer laser heating of aluminum targets in comparison with ideal blast wave theory. Appl. Surf. Sci. 127 (1998), pp. 1029-1034. 
[31] S.S. Harilal, G.V. Miloshevsky, P.K. Diwakar, N.L. LaHaye and A. Hassanein, Experimental and computational study of complex shockwave dynamics in laser ablation plumes in argon atmosphere. Phys. Plasmas 19 (2012), pp. 083504.1-083504.11.

[32] T. Pezeril, G. Saini, D. Veysset, S. Kooi, P. Fidkowski, R. Radovitzky, and K.A. Nelson, Direct visualization of laser-driven focusing shock waves. Phys. Rev. Lett. 106 (2011), pp. 214503.1-214503.4.

[33] G. Ding, C. Li, A. Zaccone, W.H. Wang, H.C. Lei, F. Jiang, Z. Ling, and M.Q. Jiang, Ultrafast extreme rejuvenation of metallic glasses by shock compression. Sci. Adv 5 (2019), pp. eaaw6249.1-eaaw6249.7.

[34] W. Soliman, T. Nakano, N. Takada, and K. Sasaki, Modification of Rayleigh-Plesset theory for reproducing dynamics of cavitation bubbles in liquid-phase laser ablation. Jpn. J. Appl. Phys. 49 (2010), pp. 116202.1-116202.6.

[35] J. Lam, J. Lombard, C. Dujardin, G. Ledoux, S. Merabia, and D. Amans, Dynamical study of bubble expansion following laser ablation in liquids. Appl. Phys. Lett. 108 (2016), pp. 074104.1-074104.5.

[36] R. Tanabe, T.T.P. Nguyen, T. Sugiura, and Y. Ito, Bubble dynamics in metal nanoparticle formation by laser ablation in liquid studied through high-speed laser stroboscopic videography. Appl. Surf. Sci. 351 (2015), pp. 327-331. 